\title{
Analyzing the Payoff of a Heterogeneous Population in the Ultimatum Game
}

\author{
Roberto da Silva and Gustavo Adolfo Kellerman \\ Instituto de Informática - UFRGS \\ Av. Bento Gonçalves, 9500, Caixa Postal 15064 \\ CEP 91501-970, Porto Alegre - RS - Brazil
}

Received on 22 May, 2007. Revised version received on 13 September, 2007

\begin{abstract}
This paper aims at showing how analytical techniques can be employed to explain the global emerged behavior of a heterogeneous population of ultimatum game players, over different strategies, by calculating their payoff moments. The ultimatum game is a game, in which two players are offered a gift to be shared. One of the players (the proposer) suggests how to divide the offer while the other player (the responder) can either agree or reject the deal. Computer simulations were performed considering the concept of turns (in every turn each participant plays necessarily only once, which is equivalent to performing matching a graph) in the game. We reproduce by simulations the expected analytical results at the limit of high number of turns. From these results, we are capable of establishing diagrams to say where each strategy is the best (optimal strategy).
\end{abstract}

Keywords: Evolutionary game theory; Ultimatum game; Payoff moments

It is very hard to find realistic models to describe the complexity of economic behavior in real societies. The same can be said about biological populations with a large number of participants over different strategies competing to survive. However, some interesting answers can be provided using Game Theory [1], which should describe negotiations among different individuals via a payoff matrix that quantifies all possible actions that can be taken by players in the game.

This theory, an accepted approach by theoretical economists due to contributions by John Nash [4] have also became accepted by biologists after the essential works of John Maynard Smith [2, 3] if we have in mind the different relations existing among species and among individuals of the same species because of a simple way different strategies can be tested and the results extrapolated to real situations in societies. In this evolutionary branch [8], this theory can bring about questions related to equilibrium, survival of strategies assuming not only one game play but several plays. Such theory is able to represent the dynamic behavior of games in average under different social contexts: in economy, such as public good games[9-11], minority game [12], in biology, prisoner dilemma [8] and others and it also can consider the different networks and their influence on the emerging collective behaviors.

In the game defined in [5], the usual ultimatum game, two players must divide a quantity between them. One of them proposes a division (the proposer) and the other can accept or reject it (the responder). If the responder rejects the offer, both players receive nothing, otherwise the "money" will be shared accordingly. A good analogy of this game, in a situation involving human beings, is how a percentage paid to an agent in a sale can be negotiated.

In human economic experiments, one could see a tendency to offer a division as near as possible to fifty-fifty and to reject values lower than $30 \%$ (see e.g. [5], [13]). However, this goes against results obtained by the classical game theory that claims participants play under Nash equilibrium [4], i.e., the rational solution is for the proposer to offer the smallest possible share, and for the responder to accept it. It is important to observe that spacial features have also been explored in the context of this game [6] as well as an analysis of the rational strategy versus a fair strategy[7], in which the player accepts values satisfying the proposer. But, as a proposer, it wants to earn compatible values (he helps and hopes to be helped).

Our goal is then to show analytically a procedure to calculate the payoff moments of players in the heterogeneous ultimatum game population according to a determined strategy. Our objective is to establish a dominant strategy for a set of input parameters. Our approach is general in the sense that all strategies are described as probability distributions and additional behaviors can be tested as different strategies. It is important to notice that other suitable games can be explored using the same kind of approach developed here. Our procedure takes into consideration the density/fractions of strategies, dominance of strategies (i.e. who is the proposer, who is the responder in different encounters) and also considers finite population corrections.

Let us consider $Y_{s_{c}}$ a random variable assuming values in $\{0,1,2, \ldots, w\}$, denoting the value obtained by a player with strategy $s_{c} \in S=\left\{s_{1}, s_{2}, \ldots, s_{p}\right\}$, where $w$ is the maximum amount of money competed (a integer variable) for a confrontation of ultimatum game, where $S$ is the set of possible strategies by players. Let us denote by $\Phi_{s_{k}}$ the density of players with strategy $s_{k}$ defined by a pair of rules (one welldefined proposal if the player turns out to be the proposer and a well-defined threshold below which the player will refuse a proposal if the player turns out to be the responder) in a population of $N$ size. Defining $\operatorname{Pr}\left(Y_{S_{c}}=i\right)$ as the probability of a received value by the player with strategy $s_{c}$ to be exactly $i$, we can then write according to law of total probability: 


$$
\begin{aligned}
\operatorname{Pr}\left(Y_{s_{c}}=i\right)= & \sum_{k=1}^{p} \operatorname{Pr}\left[\left(Y_{s_{c}}=i\right) \mid\left\{\left(s_{c} \text { is the proposer }\right) \cap\left(\text { the opponent has strategy } s_{k}\right)\right\}\right] \\
& \cdot \operatorname{Pr}\left[\left(s_{c} \text { is the proposer }\right) \mid\left(\text { the opponent has strategy } s_{k}\right)\right] \cdot \\
& \cdot \operatorname{Pr}\left(\text { the opponent has strategy } s_{k}\right) \\
& +\sum_{k=1}^{p} \operatorname{Pr}\left[\left(Y_{s_{c}}=i\right) \mid\left\{\left(s_{c} \text { is the responder }\right) \cap\left(\text { the opponent has strategy } s_{k}\right)\right\}\right] \\
& \cdot \operatorname{Pr}\left[\left(s_{c} \text { is the responder }\right) \mid\left(\text { the opponent has strategy } s_{k}\right)\right] \cdot \\
& \cdot \operatorname{Pr}\left(\text { the opponent has strategy } s_{k}\right)
\end{aligned}
$$

To describe these probabilities, we claim that there is a meaningful question concerning the existence of dominant strategies, i.e., the "ability" of a player with strategy $s_{1}$ to be the proposer/responder with probability greater than its opponent with $s_{2}$, i.e., to be proponent or responder depends not only on one's own strategy, but also on the capacity the opponent's to be the responder or proposer. Therefore we will denote: $\operatorname{Pr}\left[\left(s_{c}\right.\right.$ is the proposer $) \mid$ (the opponent has strategy $\left.\left.s_{k}\right)\right]=\rho_{s_{c}, s_{k}}=1-\operatorname{Pr}\left[\left(s_{c}\right.\right.$ is the responder $) \mid($ the opponent has strategy $\left.s_{k}\right)$ ].

But, what is the probability $\operatorname{Pr}($ the opponent has strategy $\left.s_{k}\right)$ ? This quantity depends only on the fraction of the players with strategy $s_{k}$ and if this strategy is not exactly $s_{c}$ :

$$
\operatorname{Pr}\left(\cdot \text { has strategy } s_{k}\right)=\left\{\begin{array}{cl}
\frac{\Phi_{s_{k}} N-1}{N-1} & \text { if } k=c \\
\frac{N \Phi_{s_{k}}}{N-1} & \text { if } k \neq c .
\end{array}\right.
$$

Finally, let us calculate the probabilities $\operatorname{Pr}\left(Y_{s_{c}}=i \mid s_{c}\right.$ is the proposer $\cap$ the opponent has strategy $\left.s_{k}\right)$ and $\operatorname{Pr}\left(Y_{s_{c}}=i \mid s_{c}\right.$ is the responder $\cap$ the opponent has strategy $\left.s_{k}\right)$.

Here we postulate that $\operatorname{Pr}\left(Y_{s_{c}}=i \mid s_{c}\right.$ is the proposer $\cap$ the opponent has strategy $\left.s_{k}\right)=p_{s_{c}}(i) a_{s_{k}}(w-i)$ and $\operatorname{Pr}\left(Y_{s_{c}}=i \mid s_{c}\right.$ is the acceptor $\cap$ the opponent has strategy $\left.s_{k}\right)=a_{s_{c}}(i) p_{s_{k}}(w-$ $i$ ), where $p_{\xi}(i)$ is the probability of a player with strategy $\xi \in S$ to propose the value $w-i$ to its opponent (which means $i$ for the proposer) with strategy $\zeta \in S$, and $a_{\zeta}(w-i)$ is the probability of the opponent to accept the offer.

So we have a complete probability distribution formula for a player, with strategy $s_{c}$ given by:

$$
\begin{gathered}
\operatorname{Pr}\left(Y_{s_{c}}=i\right)=p_{s_{c}}(i) \sum_{k=1}^{p} a_{s_{k}}(w-i) \frac{\Phi_{s_{k}} N-\delta_{k, c}}{N-1} \rho_{s_{c}, s_{k}}+ \\
a_{s_{c}}(i) \sum_{k=1}^{p} p_{s_{k}}(w-i) \frac{\Phi_{s_{k}} N-\delta_{k, c}}{N-1}\left(1-\rho_{s_{c}, s_{k}}\right)
\end{gathered}
$$

The $m$-th moment of the player with strategy $s_{c}$ in the population, is computed by:

$$
E\left[Y_{s_{c}}^{m}\right]=\sum_{i=0}^{w} \sum_{k=1}^{p} i^{m} \cdot p_{s_{c}}(i) a_{s_{k}}(w-i) \frac{\Phi_{s_{k}} N-\delta_{k, c}}{N-1} \rho_{s_{c}, s_{k}}+
$$

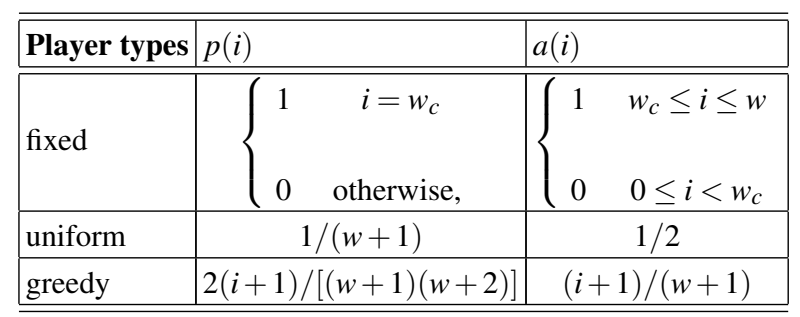

TABLE I: Players strategies.

$$
\sum_{i=0}^{w} \sum_{k=1}^{p} i^{m} \cdot a_{s_{c}}(i) p_{s_{k}}(w-i) \frac{\Phi_{s_{k}} N-\delta_{k, c}}{N-1}\left(1-\rho_{s_{c}, s_{k}}\right)
$$

From (4), considering the first moment $(m=1)$ and second moment $(m=2)$, we can calculate the dispersion (variance) of money of a player with strategy $s_{c}$ in the population:

$$
\operatorname{var}\left[Y_{s_{c}}\right]=E\left[Y_{S_{c}}^{2}\right]-E\left[Y_{S_{c}}\right]^{2}
$$

Here it is important to notice that the strategies can be absolutely general since the choice of $a_{s}$ and $p_{s}$ is arbitrary. So we can look for strategies that resemble possible interesting characteristics of participants in biological or economic populations and situations involving ambition, altruism, stubbornness and many others.

For the sake of simplicity, let us study a particular test bed $p=3$, i.e., when we have a population composed by 3 species of players (see table I): $k=1$ corresponding to a fixed payoff player (stubborn)- who wants to have the same gain, proposing always the same value $w_{c}$ and accepting values greater than $w_{c}, k=2-$ a uniform player (random), any value is proposed by it with the same probability but, as a responder, it accepts or not any value with probability $1 / 2$. Finally, $k=3$ corresponds to probabilistic greedy player (ambitious) - the probability of a value to be proposed or accepted grows linearly with the player's gain.

So, in this particular case, let us calculate the expected value of a fixed player in a population of $N$ heterogeneous players. The choice of $\rho_{s_{c}, s_{k}}$ can be very arbitrary, but for the sake of simplicity we will set $\rho_{s_{c}, s_{k}}=1 / 2$, for $k=1,2, \ldots, p$, 
i.e., there is no dominance of strategies to propose or accept.

poser functions, which leads to:

First, this result is computed, substituting the considered pro-

$$
E\left[Y_{1}\right]=\frac{1}{2}\left(w_{c} \sum_{k=1}^{3} \frac{\Phi_{s_{k}} N-\delta_{k, 1}}{N-1} a_{s_{k}}\left(w-w_{c}\right)+\sum_{k=1}^{3} \sum_{i=w_{c}}^{w} i \cdot p_{s_{k}}(w-i) \frac{\Phi_{s_{k}} N-\delta_{k, 1}}{N-1}\right)
$$

Now, it is interesting to look at each one of these sums separately. The first sum stands as:

$$
\sum_{k=1}^{3} \frac{\left(\Phi_{s_{k}} N-\delta_{k, 1}\right) a_{s_{k}}\left(w-w_{c}\right)}{N-1}=\left\{\begin{array}{l}
\frac{\Phi_{s_{1}} N-1}{N-1}+\frac{1}{2} \frac{\Phi_{s_{2}} N}{N-1}+\frac{\left(w-w_{c}+1\right)}{w+1} \frac{\Phi_{s_{3}} N}{N-1} \text { if } w_{c} \leq w / 2 \\
\frac{1}{2} \frac{\Phi_{s_{2}} N}{N-1}+\frac{\left(w-w_{c}+1\right)}{w+1} \frac{\Phi_{s_{3}} N}{N-1} \text { if } w_{c}>w / 2
\end{array}\right.
$$

and, from the second one it is easy to conclude that

$$
\begin{aligned}
\sum_{k=1}^{3} \sum_{i=w_{c}}^{w} i \cdot p_{s_{k}}(w-i) \frac{\Phi_{s_{k}} N-\delta_{k, 1}}{N-1}= & \frac{\Phi_{s_{1}} N-1}{N-1} \sum_{i=w_{c}}^{w} i \cdot p_{s_{1}}(w-i)+\frac{\Phi_{s_{2}} N}{N-1} \sum_{i=w_{c}}^{w} i \cdot p_{s_{2}}(w-i)+ \\
& +\frac{\Phi_{s_{3}} N}{N-1} \sum_{i=w_{c}}^{w} i \cdot p_{s_{3}}(w-i)
\end{aligned}
$$

Each of the above equations can be then calculated straightforwardly:

$$
\begin{aligned}
& \sum_{i=w_{c}}^{w} i \cdot p_{s_{1}}(w-i)=\left\{\begin{array}{cl}
w-w_{c} & \text { if } w_{c} \leq w / 2 \\
0 & \text { if } w_{c}>w / 2
\end{array}\right. \\
& \sum_{i=w_{c}}^{w} i \cdot p_{s_{2}}(w-i)=\sum_{i=w_{c}}^{w} \frac{i}{(w+1)}=\frac{\left(w+w_{c}\right)\left(w-w_{c}+1\right)}{2(w+1)} \\
& \sum_{i=w_{c}}^{w} i \cdot p_{s_{3}}(w-i)=\sum_{i=w_{c}}^{w} \frac{2 i(w-i+1)}{(w+1)(w+2)}=\frac{\left(w_{c}-w-1\right)\left(w_{c}-w-2\right)\left(w+2 w_{c}\right)}{3(w+1)(w+2)} .
\end{aligned}
$$

Finally, the average payoff in a population with the given characteristics can be obtained by:

$$
E\left[Y_{1}\right]= \begin{cases}\frac{1}{2} w_{c}\left[\frac{\Phi_{s_{1}} N-1}{N-1}+\frac{1}{2} \frac{\Phi_{s_{2}} N}{N-1}+\frac{\left(w-w_{c}+1\right)}{w+1} \frac{\Phi_{s_{3}} N}{N-1}\right] & \\ +\frac{1}{2}\left[\frac{\left(\Phi_{s_{1}} N-1\right)\left(w-w_{c}\right)}{N-1}+\frac{\Phi_{s_{2}} N}{N-1} \frac{\left(w+w_{c}\right)\left(w-w_{c}+1\right)}{2(w+1)}\right] & \\ +\frac{1}{2} \frac{\Phi_{s_{3}} N}{(N-1)} \frac{\left(w_{c}-w-1\right)\left(w_{c}-w-2\right)\left(w+2 w_{c}\right)}{3(w+1)(w+2)} & \text { if } w_{c} \leq w / 2 \\ \frac{1}{2} w_{c}\left[\frac{1}{2} \frac{\Phi_{s_{2}} N}{N-1}+\frac{\left(w-w_{c}+1\right)}{w+1} \frac{\Phi_{s_{3}} N}{N-1}\right]+ & \\ \frac{1}{2} \frac{\Phi_{s_{2}} N}{(N-1)} \frac{\left(w+w_{c}\right)\left(w-w_{c}+1\right)}{2(w+1)}+ & \\ \frac{1}{2} \frac{\Phi_{s_{3}} N}{N-1} \frac{\left(w_{c}-w-1\right)\left(w_{c}-w-2\right)\left(w+2 w_{c}\right)}{3(w+1)(w+2)} & \text { if } w_{c}>w / 2\end{cases}
$$

and so $E\left[Y_{1}\right]$ has a cubic polynomial dependence on $w_{c}$ for the two different branches $\left(w_{c} \leq w / 2\right.$ and $\left.w_{c}>w / 2\right)$.

To compute the variance, first of all, we need to calculate the second moment:

$$
\begin{aligned}
E\left[Y_{1}^{2}\right] & =\frac{1}{2} \sum_{i=0}^{w} \sum_{k=1}^{3} i^{2} \cdot\left[p_{s_{1}}(i) a_{s_{k}}(w-i)+a_{s_{1}}(i) p_{s_{k}}(w-i)\right] \cdot \frac{\Phi_{s_{k}} N-\delta_{k, c}}{N-1} \\
& =\frac{1}{2} w_{c}^{2} \sum_{k=1}^{3} \frac{\Phi_{s_{k}} N-\delta_{k, 1}}{N-1} a_{s_{k}}\left(w-w_{c}\right)+\frac{1}{2} \sum_{k=1}^{3} \sum_{i=w_{c}}^{w} i^{2} \cdot p_{s_{k}}(w-i) \frac{\Phi_{s_{k}} N-\delta_{k, 1}}{N-1}
\end{aligned}
$$

The first sum was computed before to calculate the average. So, calculating some necessary parts: 


$$
\begin{aligned}
& \sum_{i=w_{c}}^{w} i^{2} \cdot p_{s_{1}}(w-i)= \begin{cases}\left(w-w_{c}\right)^{2} & \text { if } w_{c} \leq w / 2 \\
0 & \text { if } w_{c}>w / 2\end{cases} \\
& \sum_{i=w_{c}}^{w} i^{2} \cdot p_{s_{2}}(w-i)=\frac{\left(w_{c}-1-w\right)\left(2 w_{c}^{2}-w_{c}+2 w_{c} w+w+2 w^{2}\right)}{6(w+1)} \\
& \sum_{i=w_{c}}^{w} i^{2} \cdot p_{s_{3}}(w-i)=\frac{\left(w_{c}-1-w\right)\left(w_{c}-w-2\right)\left(3 w_{c}^{2}-w_{c}+2 w_{c} w+w^{2}+w\right)}{6(w+1)(w+2)},
\end{aligned}
$$

we found the second moment is given by

$$
E\left[Y_{1}^{2}\right]= \begin{cases}\frac{1}{2} w_{c}^{2}\left[\frac{\Phi_{s_{1}} N-1}{N-1}+\frac{1}{2} \frac{\Phi_{s_{2}} N}{N-1}+\frac{\left(w-w_{c}+1\right)}{w+1} \frac{\Phi_{s_{3}} N}{N-1}\right] \\ +\frac{1}{2}\left[\left(w-w_{c}\right)^{2} \frac{\Phi_{s_{1}} N-1}{N-1}+\frac{\left(w_{c}-1-w\right)\left(2 w_{c}^{2}-w_{c}+2 w_{c} w+w+2 w^{2}\right)}{6(w+1)} \frac{\Phi_{s_{2}} N}{N-1}\right] \\ +\frac{\left(w_{c}-1-w\right)\left(w_{c}-w-2\right)\left(3 w_{c}^{2}-w_{c}+2 w_{c} w+w^{2}+w\right)}{12(w+1)(w+2)} \frac{\Phi_{s_{3}} N}{N-1} & \text { if } w_{c} \leq w / 2 \\ \frac{1}{2} w_{c}^{2}\left[\frac{1}{2} \frac{\Phi_{s_{1}} N}{N-1}+\frac{\left(w-w_{c}+1\right)}{w+1} \frac{\Phi_{s_{2}} N}{N-1}\right]+\frac{\left(w_{c}-1-w\right)\left(2 w_{c}^{2}-w_{c}+2 w_{c} w+w+2 w^{2}\right)}{12(w+1)} \frac{\Phi_{s_{2}} N}{N-1} & \\ +\frac{\left(w_{c}-1-w\right)\left(w_{c}-w-2\right)\left(3 w_{c}^{2}-w_{c}+2 w_{c} w+w^{2}+w\right)}{12(w+1)(w+2)} \frac{\Phi_{s_{3}} N}{N-1} & \text { if } w_{c}>w / 2\end{cases}
$$

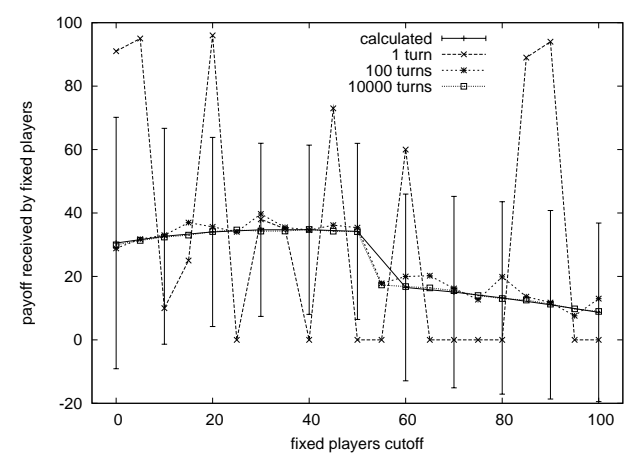

FIG. 1: The calculated value of average fixed player payoff $E\left[Y_{1}\right]$ with the corresponding standard deviation $\sqrt{\operatorname{var}\left[Y_{1}\right]}$, compared to the mean value received by one fixed player in different numbers of turns.

For a comparison, we now present several computer simulations. These simulations use the concept of turn [14] i.e., for each iteration a pair of players is chosen randomly, but all players take part only once (this is formally known in data structure as "matching") as described by algorithm 1. For example, in a population of 200 players, in every turn 100 pairs are randomly arranged. For our experiments we assume $w=100$.

Figure 1 shows the received payoff $E\left[Y_{1}\right]$ (fixed players) obtained analytically by equation 10 (continuous curve). The population has the same proportion of fixed, uniform and greedy players (1/3). One can notice that for a high number of turns, the experimental results agree with the analytical ones. This agreement is not observed in a low number of turns $\left(N_{t}<100\right)$, which is natural according to "analytical

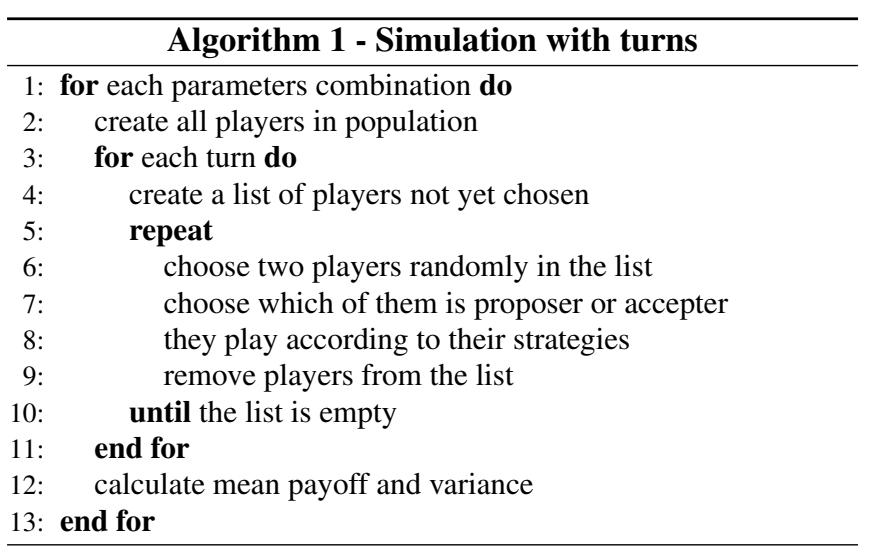

error bars" $\left(\sqrt{\operatorname{var}\left[Y_{1}\right]}\right)$ according to equations 13 and 10 .

The expected crossover in equation 10 can be observed exactly in $w_{c}=50(w=100)$. The payoff of the uniform and greedy players according to the cutoff of fixed players can also be analyzed numerically calculating the average directly from equation 4, i.e., performing the sums of this equation (see Fig. 2) and not obtaining exactly an analytical expression like 10 . In this case, we have also considered simulation experiments simultaneously for a comparison, studying the effects of an increase in the number of turns observing, like in Fig. 1, that the results of simulation are the same as the exact formulae for $N_{t} \sim 1000$, in the exact sense of law of large numbers (see figure 2). In this case we observe a continuous decay of payoff for both players (greedy and uniform) as a function of fixed players cutoff, different from the observed discontinuity that occurs for fixed players (Fig. 1).

Finally, we would like to analyze the dominance regions of 

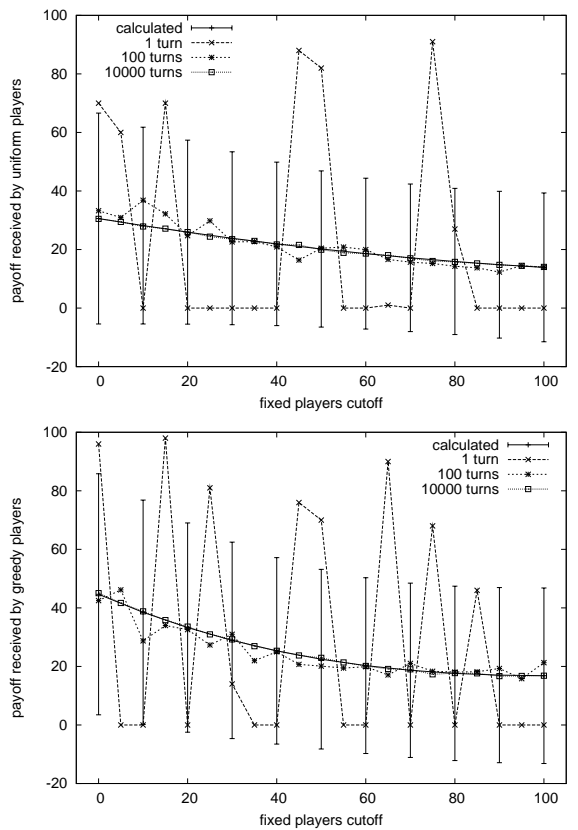

FIG. 2: The average payoff of greedy and uniform players in a population with the same proportion (1/3) of players: fixed, greedy and uniform. In both cases we observe a continuous decay as function of fixed players cutoff.
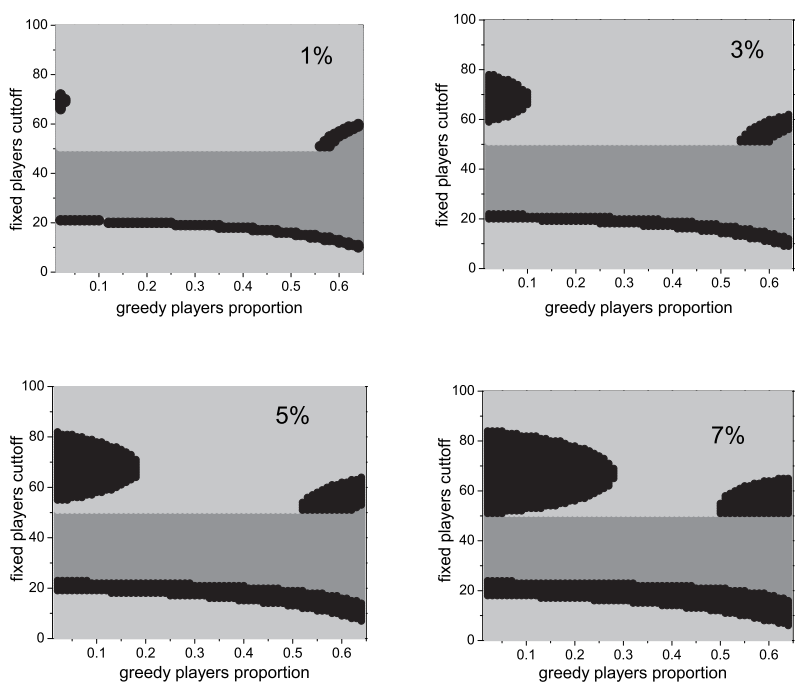

FIG. 3: Dominance of strategies fixing the proportion of uniform players at $1 / 3$. The different plots correspond to the different draw criteria respectively.

players based on the average payoff, i.e., who has the best strategy changing the proportion of greedy players and the cutoff of fixed ones. For this experiment, we have fixed the proportion of uniform players at $1 / 3$, and we changed the greedy proportion keeping the complement of population with fixed players.

In Fig. 3, the sequence of plots shows a diagram of strate-
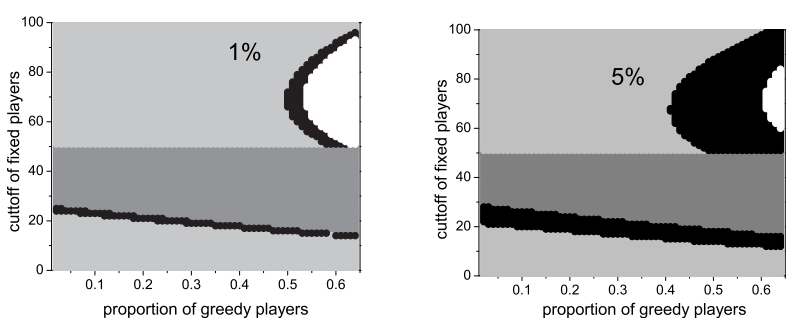

FIG. 4: Dominance of strategies fixing the proportion of the fixed players at $1 / 3$. We considered $1 \%$ (left side) and 5\% (right side) as draw criteria respectively.
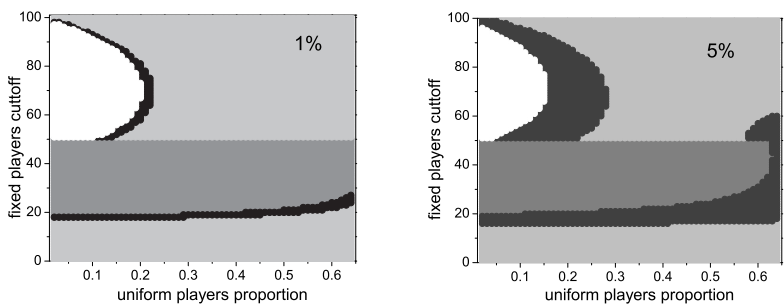

FIG. 5: Plot similar to figure 4 but fixing $1 / 3$ of greedy players in the population.

gies looking at dominance of different players/strategies. The light gray color corresponds to a region where the greedy player has the highest average payoff while the dark gray corresponds to a dominant region for fixed players. The black dots represent a region where a draw occurs among players considering different draw criteria, errors (absolute differences among the payoffs) of $1 \%, 3 \%, 5 \%$ and $7 \%$ respectively.

We can observe an increase in draw regions as a result of relaxation of draw criteria. Considering this particular configuration, there is no region where the uniform players win, showing this strategy (play roulette, play coin) to negotiate is not so good to ultimatum game if the population is composed just by $1 / 3$ of uniform players independently of cutoff used by fixed players, not even in the case where the strategies are equally distributed $(1 / 3,1 / 3,1 / 3)$ in the population.

Additionally, we perform two similar alternative simulations: firstly, fixing the proportion of fixed players at $1 / 3$ (see Fig. 4) and secondly, fixing the proportion of greedy players at $1 / 3$ (figure 5).

When the uniform players are not fixed at $1 / 3$ in the population, regions where they obtain the highest payoff are found (represented by white regions in Figs. 4 and 5, the other tones follow the same definition of Fig. 3). These regions become smaller for more relaxed draw criteria losing area for draw regions (although only $1 \%$ and $5 \%$ are shown here, the other cases were simulated by authors and corroborate this trend).

It is important to notice the general aspect of these results regarding the several strategies that can be adopted and the different extensions to be explored to cover additional different games in an evolutionary context. 


\section{Acknowledgments}

R. da Silva thanks CNPq for the financial support to this work.
[1] J. Von Neumann, O. Morgenstern, Theory of Games and Economic Behavior, Princeton University Press (1953).

[2] J. M. Smith, G. R. Price, Nature 246, 15 (1973).

[3] J. M. Smith, Evolution and the Theory of Games, Cambridge University Press (1982)

[4] J. Nash, Proceedings of Nacional Academy of Science of the United States of America 36, 48 (1950).

[5] W. Guth, R. Schmittberger, B. Schwarze, Journal of Economic Behavior and Organization 3(4), 367 (1982).

[6] K. M. Page, M. A. Nowak, and K. Sigmund, Proc. R. Soc. Lond. B 267, 2177 (2000).

[7] M. A. Nowak, K. Page, and K. Sigmund, Science 289, 1773 (2000).

[8] G. Szäbó, G. Fáth, Phys. Rep. 446, 97 (2007).

[9] G. Szabó, C. Hauert, Physical Review Letters 89(11) 118101:1-

$$
4 \text { (2002). }
$$

[10] R. da Silva, A.L.C. Bazzan, A.T. Baraviera, and S.R. Dahmen, Physica A 371, 610 (2006).

[11] R. da Silva, A.T. Baraviera, S.R. Dahmen, and A.L.C. Bazzan, Lecture Notes in Economic and Mathematical Systems 584, 221 (2006).

[12] D. Challet and Y. C. Zhang, Physica A 246, 407 (1997).

[13] J. Henrich, R. McElreath, A. Barr, J. Esminger, C. Barret, A. Bolyanatz, J.C. Cardenas, M. Gurven, E. Gwako, N. Henrich, C. Lesorogol, F. Marlowe, D. Tracer, and J. Ziker, Science 312(5781), 1767 (2006).

[14] G. A. Kellerman, R. da Silva, submitted to Journal of Theoretical Biology (2007). 